\title{
An enhanced recovery after surgery program for hip and knee arthroplasty
}

\author{
The ERAS \\ program had \\ a small but \\ significant \\ effect on \\ hospital stay, \\ particularly \\ for knee \\ replacement \\ patients
}

\section{Nicholas Christelis FFPMRCA, FANZCA FFPMANZCA, \\ Sophie Wallace BHSc(Nurs) GradCertlnfectCont, MPH'}

Claire E Sage

BAppSci(Nurs), MNurs

Uate Babitu

MBBS, FANZCA

Susan Liew MBBS(Hons), FRACS(Orth)

James Dugal MBBS, FRACS ${ }^{3}$

Ibolya Nyulas MSc, GradDipMgt ${ }^{1.2}$

Nora Mutalima BSc, MBChB, DPhil ${ }^{4}$

Ton Tran MBBS, FRACS, FAOA

Paul S Myles MD, FRARCSI, FANZCA'

1 The Alfred Hospital, Melbourne, VIC

2 Monash University, Melbourne, VIC.

3 Bendigo Hospital, Bendigo, VIC.

4 Dandenong Hospital, Melbourne, VIC.

5 Monash Health, Melbourne, VIC.

n.christelis@ alfred.org.au

doi: 10.5694/mjal4.00601

Abstract

Objective: To institute and evaluate the benefits of an enhanced recovery after surgery (ERAS) program across three hospitals in Victoria.

Design, setting and participants: We used a before-and-after quality improvement study design consisting of three phases: pre-ERAS program data collection from March to September 2012; ERAS training and implementation during September 2012; and change performance measurement following ERAS implementation from October 2012 to May 2013.

Main outcome measures: The primary end point was duration of hospital stay after knee or hip arthroplasty. Secondary end points were adherence to the ERAS bundle, and process and patient recovery characteristics.

Results: We enrolled 412 patients to the pre-ERAS (existing-practice) phase and compared them with 297 patients in the ERAS phase. For ERAS patients, compared with existing-practice patients, hospital stay was reduced (geometric mean, 5.3 [SD, 1.6] v 4.9 [SD, 1.6] days; $P<0.001$ ) and there was a significant improvement in the proportion of patients ready for discharge on Day 3 after surgery (41\% v 59\%; $P<0.001$ ). The most common reason for delayed discharge was patients waiting for review or access to rehabilitation services. There were markedly improved indicators of processes and outcomes of care, including improved patient education, reduced fasting times, less blood loss, better analgesia, earlier ambulation and improved overall quality of recovery.

Conclusion: We found that an ERAS program could be successfully implemented in elective joint arthroplasty, leading to a shorter duration of hospital stay. We recommend this orthopaedic ERAS pathway.

perioperative care (the fast-track methodology) reduces the need for prolonged hospitalisation and convalescence, and reduces morbidity, with subsequent economic savings. ${ }^{2-6}$ Enhanced recovery after surgery (ERAS) programs are a care package of evidence-based interventions used in a multimodal, integrated clinical care pathway to achieve improved functional outcomes and rapid recovery. ${ }^{6}$ ERAS pathways have led to reduced hospital stays after hip or knee replacements - as short as 3 days in many centres. $^{5-8}$ of individual care components in

1 Sixteen predefined enhanced recovery after surgery items for hip or knee arthroplasty

- Nurse coordinator counselling in the orthopaedic or preadmission clinic

- Preadmission review by a physiotherapist and/or dietitian

- Minimal fasting preoperatively, defined as clear oral fluids up to 2 hours before surgery

- Preoperative oral carbohydrate loading

- No sedative premedication (benzodiazepines, opioids or neuroleptics)

- Pre-emptive analgesia with paracetamol and gabapentinoids according to protocols

- Spinal anaesthesia (not epidural)

- Local anaesthesia technique (surgeon-delivered local infiltration of analgesia or anaesthetic femoral nerve block)

- Minimal ( $\leqslant 10 \mathrm{mg}$ ) intravenous morphine intraoperatively

- Intraoperative avoidance of excessive intravenous fluids (knee, >1L; hip, > 2L; both: subtracting blood loss)

- Active intraoperative warming (forced air warming and/or warmed intravenous fluids)

- Antiemetic prophylaxis

- Multimodal oral analgesia for $\geqslant 3$ days postoperatively, to include a non-steroidal anti-inflammatory drug or cyclooxygenase-2 inhibitor

- Early postoperative (recovery room) oral carbohydrate supplementation

- Mobilisation within 24 hours

- Early hospital discharge ( $\leqslant 5$ days) 


\begin{tabular}{|c|c|c|c|}
\hline Variable & $\begin{array}{l}\text { Existing practice } \\
\quad(n=412)\end{array}$ & $\begin{array}{c}\text { ERAS } \\
(n=297)\end{array}$ & $P$ \\
\hline Sex, male & $164(40 \%)$ & $113(38 \%)$ & 0.64 \\
\hline Mean age, years (SD) & $68(11)$ & $67(10)$ & 0.22 \\
\hline Mean weight, kg (SD) & $84(19)$ & $87(20)$ & 0.092 \\
\hline \multicolumn{4}{|l|}{ Medical history } \\
\hline Current smoker & $46(11 \%)$ & $30(10 \%)$ & 0.65 \\
\hline Hypertension & $284(69 \%)$ & $194(65 \%)$ & 0.31 \\
\hline Coronary artery disease & $70(17 \%)$ & $41(14 \%)$ & 0.25 \\
\hline Stroke & $20(5 \%)$ & $10(3 \%)$ & 0.33 \\
\hline Heart failure & $19(5 \%)$ & $15(5 \%)$ & 0.86 \\
\hline Peripheral vascular disease & $13(3 \%)$ & $8(3 \%)$ & 0.72 \\
\hline Diabetes & $81(20 \%)$ & $73(25 \%)$ & 0.12 \\
\hline COPD & $88(21 \%)$ & $67(23 \%)$ & 0.70 \\
\hline Preoperative anaemia & $36(9 \%)$ & $22(7 \%)$ & 0.52 \\
\hline \multicolumn{4}{|l|}{ Usual medications } \\
\hline Opioid & $106(26 \%)$ & $58(20 \%)$ & 0.053 \\
\hline Aspirin within 5 days & $58(14 \%)$ & $54(18 \%)$ & 0.14 \\
\hline Clopidogrel within 7 days & $2(<1 \%)$ & 0 & 0.23 \\
\hline Warfarin within 7 days & $22(5 \%)$ & $6(2 \%)$ & 0.025 \\
\hline NSAID/COX-2 inhibitor & $109(26 \%)$ & $109(37 \%)$ & 0.004 \\
\hline ACE inhibitor/ARB & 235 (57\%) & $168(57 \%)$ & 0.90 \\
\hline Beta blocker & $71(17 \%)$ & $51(17 \%)$ & 0.98 \\
\hline Statin & $141(34 \%)$ & 89 (30\%) & 0.37 \\
\hline Calcium channel blocker & $98(24 \%)$ & $73(25 \%)$ & 0.81 \\
\hline Diuretic & $120(29 \%)$ & 79 (27\%) & 0.45 \\
\hline Oral hypoglycaemic & $57(14 \%)$ & $60(20 \%)$ & 0.024 \\
\hline Insulin & $9(2 \%)$ & $11(4 \%)$ & 0.25 \\
\hline LMWH & $20(5 \%)$ & $8(3 \%)$ & 0.15 \\
\hline ASA physical status & & & $0.57^{\dagger}$ \\
\hline 1 & $10(2 \%)$ & $10(3 \%)$ & \\
\hline 2 & $223(54 \%)$ & $161(54 \%)$ & \\
\hline 3 & $172(42 \%)$ & $122(41 \%)$ & \\
\hline 4 & $7(2 \%)$ & $3(1 \%)$ & \\
\hline Disease & & & 0.22 \\
\hline Osteoarthritis & 367 (89\%) & $275(93 \%)$ & \\
\hline Rheumatoid arthritis & $4(1 \%)$ & 0 & \\
\hline Avascular necrosis & $14(3 \%)$ & $7(2 \%)$ & \\
\hline Other & $27(7 \%)$ & $15(5 \%)$ & \\
\hline Previous PONV or motion sickness & $124(30 \%)$ & 81 (27\%) & 0.41 \\
\hline
\end{tabular}

$\mathrm{ACE}$ = angiotensin-converting enzyme. $\mathrm{ARB}=$ angiotensin-receptor blocker. $\mathrm{ASA}=$ American Society of Anesthesiologists. COPD = chronic obstructive pulmonary disease.

COX = cyclooxygenase. ERAS = enhanced recovery after surgery, $\mathrm{LMWH}=$ low molecular weight heparin. NSAID = non-steroidal anti-inflammatory drug. PONV = postoperative nausea and vomiting. * Data are no. (\%) of patients unless otherwise specified. $\uparrow P$ value derived from $\chi^{2}$ test for trend.

We aimed to assess the extent to which a predefined ERAS program for orthopaedic surgical patients could be achieved, and to evaluate improvements in quality of care and patient outcome across three public hospitals in Victoria.
Phase 1: over the 6 months before implementation of the ERAS program, we recorded perioperative data for all eligible patients undergoing surgery (the existing-practice cohort).

Phase 2: training of staff managing orthopaedic surgical patients. For 1 month, the evidence-based background to ERAS was promulgated to all surgical, anaesthetic and nursing staff. This was done in various forms including lectures, workshops, meetings and written instructions.

Phase 3: change performance. We undertook a repeat audit following the implementation of the ERAS care package (the ERAS cohort).

Our study received ethics approval as an audit project with a waiver for specific patient consent (Alfred Human Research Ethics Committee, EC 92/12).

The pre-ERAS phase ran from March to September 2012. Training of staff took place over September 2012. The ERAS phase ran from October 2012 to May 2013.

Patient health status was quantified using the American Society of Anesthesiologists physical status classification, ranging from 1 (healthy patient) to 5 (moribund patient not expected to survive without the operation). Patient quality of recovery was assessed using the patientcentred, 15-item quality-of-recovery score, ${ }^{9}$ and the 12-item World Health Organization Disability Assessment Schedule 2.0 score. $^{10}$ Indicators of patient satisfaction were assessed at 30 days after surgery using a 5 -item Likert scale $(0=$ strongly agree, $5=$ strongly disagree).

Actual hospital stay was timed from the beginning of surgery until discharge. We evaluated readiness for discharge on postoperative Day 3, defined by whether patients were eating and drinking, had no drain tubes or urinary catheters, were weightbearing, and had well controlled pain scores (visual analogue scale with a range of 0 to 10) at rest and on movement of less than 3 and 5, respectively.

A successful ERAS implementation required at least 11 of 16 prespecified ERAS items (Box 1). 
On completion of our study, there was a concern raised by the surgical team at the lead institution regarding an apparent increased incidence of acute kidney injury (AKI). In view of the widespread use of local anaesthetic infiltration with a solution that included ketorolac $30 \mathrm{mg}$, we chose to investigate this more formally at the lead institution. We retrospectively retrieved all perioperative creatinine data for the study cohorts. AKI was defined according to AKI Network ${ }^{11}$ and RIFLE (risk, injury, failure, loss, end-stage kidney disease) $)^{12}$ criteria. We did not include urine output or oliguria in the definitions of AKI, in part because we did not collect these data, but primarily because urine output is an unreliable indicator of renal function in the perioperative setting.

To account for the restrictive intravenous (IV) fluid regimen used in the ERAS cohort (which may have artificially elevated serum creatinine because of the avoidance of a dilutional effect from excessive IV fluids increasing body water), we calculated the adjusted creatinine concentration by first estimating the volume of distribution for creatinine as equal to total body water (assumed to be $60 \%$ of body weight, expressed in $\mathrm{mL}$ ), and assuming that $50 \%$ of IV fluid was still accumulated as tissue oedema at the time of postoperative creatinine measurements: ${ }^{13}$

adjusted creatinine concentration $=$ serum creatinine concentration $\times(1+[0.5 \times$ IV fluid balance/total body water])

Analysis of the data showed that there was no increased incidence of AKI in the ERAS group (Appendix 1).

The primary end point of the study was duration of hospital stay. Secondary end points were adherence to the ERAS bundle (defined as $\geqslant 11$ items), and a number of patient outcome measures. A sample size calculation based on a change in hospital stay from a mean of 7 days (SD, 4 days) to 6 days (SD, 3 days), with an $\alpha$ value of 0.05 and a $\beta$ value of 0.2 , required at least 380 patients to be enrolled, but we included a larger sample in view of the planned subgroup analyses. Continuous data are

\section{Perioperative and surgical care*}

\begin{tabular}{|c|c|c|c|}
\hline Variable & $\begin{array}{l}\text { Existing practice } \\
\quad(n=412)\end{array}$ & ERAS $(n=297)$ & $P$ \\
\hline \multicolumn{4}{|l|}{ Preadmission clinic, seen by: } \\
\hline Nurse $^{\dagger}$ & $406(99 \%)$ & $297(100 \%)$ & 0.037 \\
\hline Anaesthetist & 405 (98\%) & $297(100 \%)$ & 0.024 \\
\hline Surgeon & $406(99 \%)$ & $297(100 \%)$ & 0.037 \\
\hline Physiotherapist $^{\dagger}$ & $331(80 \%)$ & $224(75 \%)$ & 0.12 \\
\hline Occupational therapist & $324(79 \%)$ & $249(84 \%)$ & 0.077 \\
\hline Dietitian $^{\dagger}$ & 0 & $61(21 \%)$ & $<0.001$ \\
\hline \multicolumn{4}{|l|}{ Day of surgery } \\
\hline Admission on day of surgery & $404(98 \%)$ & $294(99 \%)$ & 0.32 \\
\hline Shower with antibiotic soap & $399(97 \%)$ & $238(80 \%)$ & $<0.001$ \\
\hline Preoperative skin wipes & $4(1 \%)$ & $91(31 \%)$ & $<0.001$ \\
\hline Oral (clear) fluids given ${ }^{\dagger}$ & $2(<1 \%)$ & $180(61 \%)$ & $<0.001$ \\
\hline Oral carbohydrate drink ${ }^{\dagger}$ & 0 & $248(84 \%)$ & $<0.001$ \\
\hline Gabapentin premedication ${ }^{\dagger}$ & $21(5 \%)$ & $172(58 \%)$ & $<0.001$ \\
\hline \multicolumn{4}{|l|}{ Type of surgery } \\
\hline Hip & $214(52 \%)$ & $129(43 \%)$ & 0.025 \\
\hline Knee & $198(48 \%)$ & $168(57 \%)$ & 0.025 \\
\hline Revision & $26(6 \%)$ & $13(4 \%)$ & 0.26 \\
\hline \multicolumn{4}{|l|}{ Tubes } \\
\hline Urinary catheter & $200(49 \%)$ & $107(36 \%)$ & 0.001 \\
\hline Drain tube(s) & $105(25 \%)$ & $59(20 \%)$ & 0.080 \\
\hline \multicolumn{4}{|l|}{ Type of anaesthesia } \\
\hline General ( \pm regional) & $266(65 \%)$ & $164(55 \%)$ & 0.014 \\
\hline Spinal $^{\dagger}$ & $236(57 \%)$ & 205 (69\%) & 0.001 \\
\hline Epidural or CSE & $16(4 \%)$ & $3(1 \%)$ & 0.019 \\
\hline \multicolumn{4}{|l|}{ Postoperative regional analgesia } \\
\hline Nerve block used & $135(33 \%)$ & $44(15 \%)$ & $<0.001$ \\
\hline LA infiltration $^{\dagger}$ & $214(52 \%)$ & $222(75 \%)$ & $<0.001$ \\
\hline PONV prophylaxis $^{\dagger}$ & $233(57 \%)$ & $202(68 \%)$ & 0.002 \\
\hline Mean total IV fluids, $\mathrm{mL}(\mathrm{SD})^{\dagger}$ & $1756(767)$ & $1446(687)$ & $<0.001$ \\
\hline Active (forced air) warming ${ }^{\dagger}$ & $392(95 \%)$ & $285(96 \%)$ & 0.34 \\
\hline Mean lowest temperature, ${ }^{\circ} \mathrm{C}(\mathrm{SD})$ & $35.7(0.5)$ & $36.2(0.4)$ & 0.039 \\
\hline Mean duration of surgery, hours (SD) & $2.0(0.9)$ & $1.9(0.6)$ & 0.33 \\
\hline
\end{tabular}

CSE $=$ combined spinal and epidural. ERAS $=$ enhanced recovery after surgery. IV $=$ intravenous. $L A=$ local anaesthesia. PONV = postoperative nausea and vomiting. * Data are no. (\%) of patients unless otherwise specified. $†$ Key ERAS implementation points.

reported as mean (SD) or median and interquartile range (IQR). Numerical data were first tested for normality using the Kolmogorov-Smirnov test and then compared using the Student $t$ test or Wilcoxon rank-sum test, as appropriate. Rates were compared using $\chi^{2}$ or Fisher exact test, as appropriate. Hospital stay was expected to be skewed to the right because of a small proportion experiencing complications and a protracted hospital stay. Therefore, we log-transformed hospital stay data to enable valid comparison using the $t$ test; in addition, we report median (IQR) length of stay and results of Wilcoxon rank-sum testing. Patients undergoing each type of surgery were also analysed as subgroups. A $P$ value of less than 0.05 was considered statistically significant. Data were analysed with SPSS version 20.0 for Windows (SPSS Inc). 
4 Recovery profile and hospital stay

\begin{tabular}{|c|c|c|c|}
\hline Variable & $\begin{array}{l}\text { Existing practice } \\
\qquad(n=412)\end{array}$ & $\begin{array}{c}\text { ERAS } \\
(n=297)\end{array}$ & $P$ \\
\hline \multicolumn{4}{|l|}{ Recovery room } \\
\hline Median pain score (IQR), at rest* & $0(0-5)$ & $0(0-4)$ & 0.047 \\
\hline Median pain score (IQR), on movement* & $0(0-7)$ & $0(0-4)$ & $<0.001$ \\
\hline Admission temperature, ${ }^{\circ} \mathrm{C}(\mathrm{SD})$ & $36.1(0.5)$ & $36.0(0.6)$ & 0.006 \\
\hline \multicolumn{4}{|l|}{ Postoperative, at 24 hours } \\
\hline Median pain score (IQR), at rest* & $5(3-7)$ & $4(2-5)$ & $<0.001$ \\
\hline Median pain score (IQR), on movement* & $6(4-8)$ & $5(3-7)$ & $<0.001$ \\
\hline Mean quality of recovery score (SD) (range, 0-150) & $103(19)$ & $106(20)$ & 0.056 \\
\hline \multicolumn{4}{|l|}{ Total knee replacement } \\
\hline Mean knee flexion (SD), degrees & $51(19)$ & $57(24)$ & 0.026 \\
\hline Median quadriceps strength (IQR), Nm & $3(2-3)$ & $2(2-3)$ & 0.11 \\
\hline \multicolumn{4}{|l|}{ Postoperative, at 48 hours } \\
\hline Median pain score (IQR), at rest* & $4(2-6)$ & $3(1-5)$ & $<0.001$ \\
\hline Median pain score (IQR), on movement* & $6(4-8)$ & $5(2-7)$ & 0.001 \\
\hline \multicolumn{4}{|l|}{ Total knee replacement } \\
\hline Mean knee flexion (SD), degrees & $72(19)$ & $78(14)$ & 0.009 \\
\hline Median quadriceps strength (IQR), Nm & $3(2-3)$ & $3(2-3)$ & 0.90 \\
\hline \multicolumn{4}{|l|}{ Recovery parameters, median hours (IQR) } \\
\hline Weight bearing & $1.1(1.0-2.0)$ & $1.0(0.9-2.0)$ & 0.001 \\
\hline Oral fluid intake & $3.2(2.0-5.0)$ & $2.7(1.7-4.1)$ & 0.016 \\
\hline Oral food intake & $7.0(4.3-15)$ & $6.3(3.2-7.9)$ & 0.004 \\
\hline Removal of drain tubes & $27(24-42)$ & $25(23-27)$ & 0.002 \\
\hline Removal of urinary catheter & $48(42-76)$ & $33(17-60)$ & $<0.001$ \\
\hline Blood transfusion in hospital, no. of patients (\%) & $58(14 \%)$ & $31(10 \%)$ & 0.24 \\
\hline Return to theatre, no. of patients (\%) & $14(3 \%)$ & $10(3 \%)$ & 0.76 \\
\hline \multicolumn{4}{|l|}{ Length of stay, days } \\
\hline Geometric mean (SD) & $5.3(1.6)$ & $4.9(1.6)$ & $<0.001$ \\
\hline Median (IQR) & $5.0(4.0-6.8)$ & $5.0(3.8-6.2)$ & 0.10 \\
\hline
\end{tabular}

ERAS $=$ enhanced recovery after surgery. $\mathrm{IQR}=$ interquartile range. $*$ Visual analogue scale: $0=$ none, $10=$ severe. surgery, the ERAS program was associated with a reduced hospital stay (geometric mean, $5.3[S D, 1.6] \mathrm{v}$ $4.5[\mathrm{SD}, 1.5]$ days $[P=0.001] ;$ median, 5.0 [IQR, 4.0-6.7] v 4.1 [IQR, 3.0-6.0] days $[P=0.005])$; and a greater proportion of patients were more likely to be discharged by Day 5 (64\% v 52\%; $P=0.019)$. There was no change in median hospital stay for hip replacement patients in the ERAS group compared with the existing-practice group (median, 5.0 [IQR, 3.5-7.0] v 5.0 [IQR, 4.0-6.9] days; $P=0.99$ ). Overall, the 75th centile for length of stay decreased from 6.8 to 6.0 days.

We found high rates of compliance with nearly all ERAS items (Box 3). There was increased use of spinal anaesthesia. The use of femoral nerve block (with or without a catheter) was substituted by favouring surgeondelivered local anaesthetic infiltration in $75 \%$ of cases; this change in practice varied across the three hospitals (98\%, 37\% and 98\%). There were improved dynamic pain scores and quality of recovery (Box 4). There were improvements in other recovery parameters (early feeding, ambulation and removal of tubes). Patients undergoing knee replacement had improved flexion on postoperative Days 1 and 2.

The proportion of patients ready for discharge on Day 3 after surgery was significantly higher in the ERAS group compared with the existing-practice group: $59 \%$ v $41 \%$, respectively; relative risk, 1.35 (95\% CI, 1.18-1.53); $P<0.001$ (Box 6 and Appendix 1).

The 6-week complication rates were similar and there was no increase in the rate of hospital readmission. Pain levels were similar and there was a higher level of patient satisfaction at 6 weeks after surgery (Box 5).

The incidence of AKI was comparable between groups (Appendix 1). The final plasma creatinine values were slightly higher in the ERAS group, but this could be accounted for by higher baseline (preoperative) values; ERAS patients had a mean change in creatinine from $78 \mathrm{mmol} / \mathrm{L}$ (SD, $24 \mathrm{mmol} / \mathrm{L}$ ) preoperatively, to a final reading of $79 \mathrm{mmol} / \mathrm{L}(\mathrm{SD}$, 
$24 \mathrm{mmol} / \mathrm{L})$ postoperatively. There were no cases of renal failure.

\section{Discussion}

Our study results indicate that implementing an ERAS program may be beneficial for other Victorian public hospitals. The ERAS program had a small but significant effect on hospital stay, particularly for knee replacement patients. A pertinent finding from our study was that a higher proportion of patients managed through the ERAS care pathway compared with the existing-practice group (59\% v 41\%, respectively) were deemed ready for discharge on postoperative Day 3.

The limited effect on actual hospital stay in this project is likely to be due to one key factor: despite an effectively implemented ERAS program, there were entrenched hospital practices that prevented earlier hospital discharge even though patients were deemed ready for discharge; that is, discharge planning practice was mostly unchanged. This is due to established ward practices, including a repetitive requirement for many joint replacement patients to undergo their initial rehabilitation program as an inpatient (delaying their discharge). Patients referred for rehabilitation often wait for some time (hours or days) before being reviewed by rehabilitation services. Further, if surgery occurred on a Thursday or Friday, patients had minimal access to physiotherapy over the weekend. These aspects offer opportunities for improvement. The challenge is clear: to convert improvement in care (and outcome) into shorter hospital stay.

Our primary end point was duration of hospital stay. As we have done previously, ${ }^{14}$ we used a logtransformation and compared geometric means to account for skewness of our data (a small proportion of patients staying in hospital for very long times distorts central tendency - a well known phenomenon for many types of surgery). A secondary non-parametric comparison of median stays was not statistically significant. Therefore, we reported the 75th centiles to illustrate the observed

\begin{tabular}{lccc}
5 Recovery profile at 6 weeks after surgery* & & \\
Variable & $\begin{array}{c}\text { Existing practice } \\
(n=412)\end{array}$ & $\begin{array}{c}\text { ERAS } \\
(n=297)\end{array}$ & $P$ \\
\hline Wound infection & $21(5 \%)$ & $13(4 \%)$ & 0.99 \\
Prosthesis infection & $5(1 \%)$ & $2(<1 \%)$ & 0.60 \\
Prosthetic joint dislocation & $2(<1 \%)$ & $3(1 \%)$ & 0.31 \\
Periprosthetic fracture & 0 & 0 & $>0.99$ \\
Thromboembolism & $13(3 \%)$ & $10(3 \%)$ & 0.59 \\
Urinary tract infection & $8(2 \%)$ & $2(1 \%)$ & 0.22 \\
Death & $2(<1 \%)$ & $1(<1 \%)$ & 0.85 \\
Worst pain rating in past 24 hours ${ }^{\dagger}$ & $2(0-4)$ & $2(0-3)$ & 0.01 \\
Extent of disability in past 24 hours ${ }^{\dagger}$ & $2(0-3)$ & $1(0-2)$ & 0.37 \\
Patient satisfaction ${ }^{\ddagger}$ & & & $<0.001$ \\
$\quad$ Was surgery worthwhile? & $1(1-2)$ & $1(1-1)$ & 0.015 \\
\multicolumn{1}{l}{ Did surgery improve your daily life? } & $1(1-2)$ & $1(1-2)$ & $<0.001$ \\
Do you feel better? & $1(1-2)$ & $1(1-2)$ & 0.67 \\
Do you have trouble sleeping? & $3(2-4)$ & $3(2-4)$ & 0.87 \\
Hospital readmission & $25(6 \%)$ & $15(5 \%)$ & \\
\hline
\end{tabular}

ERAS $=$ enhanced recovery after surgery. $*$ Data are no. (\%) of patients or median score (IQR). $†$ Visual analogue scale: $0=$ none, 10 = severe. $\neq 5$-point Likert scale: 0 = strongly agree, 5 = strongly disagree.

improvement in hospital stay for the majority of patients.

Administrative and traditional patterns of clinical practice limit opportunities for change and are common causes of delayed discharge from hospital. ${ }^{14-16}$ Perhaps specific fasttrack arthroplasty units that have evidence-based and protocolised rapid recovery pathways can optimise cost-efficient quality outcomes after hip and knee replacement surgery. ${ }^{15}$ This could reduce hospital costs, improve patient satisfaction with care and potentially reduce perioperative morbidity.

We demonstrated that an ERAS program for orthopaedic joint replacement can be achieved. We markedly improved most indicators of processes related to an ERAS program. These included preadmission patient education, reduced fasting times, clear oral fluids, written instructions (including expected day of discharge), less blood loss, better pain relief, earlier ambulation and better overall quality of recovery. Similar success has been reported in other countries. $^{8,17,18}$ Medical teams can be trained to deliver an ERAS program and this clearly improves the quality of care.

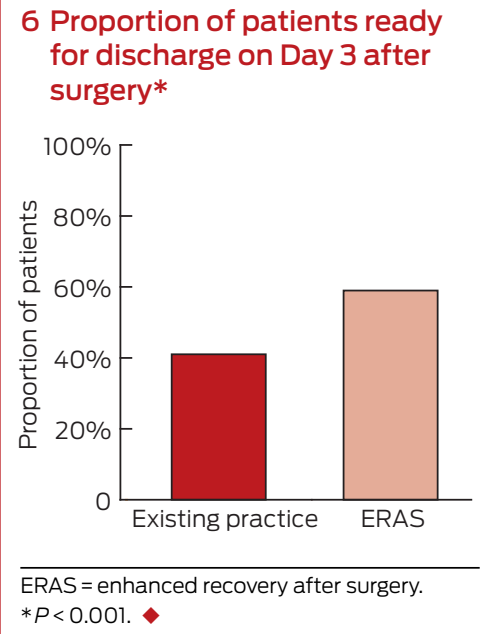

We clearly demonstrated that we could successfully implement a predefined ERAS program for orthopaedic surgical patients in public hospitals, and that doctors and nurses could follow such a regimen to improve outcome parameters. There was high uptake of nearly all ERAS items. This led to clinically important improvements in care, a small reduction in hospital stay for knee replacement patients, and an overall improvement in some aspects of patient satisfaction. There was evidence of improved quality of recovery. There was no effect on most 
complications and no adverse effect on hospital readmission rates.

Our 6-week complication rates are lower than those reported in most studies, the largest of which included 4500 consecutive unselected hip and knee replacements. ${ }^{5}$ In that study, the first 3000 patients represented existing or traditional practice and a further 1500 patients underwent an ERAS protocol similar to that used in our study. This group reported a decrease in length of stay from 6 days to 3 days $(P<0.001)$, as well as a reduction in 30-day mortality (from $0.5 \%$ to $0.1 \% ; P=0.02$ ) and 90 -day mortality (from $0.8 \%$ to $0.2 \% ; P=0.01$ ). Blood transfusion was reduced from $23 \%$ to $9.8 \%(P<0.001)$. There was a trend of a reduced rate of 30-day myocardial infarction (from $0.8 \%$ to $0.5 \% ; P=0.2$ ) and stroke (from $0.5 \%$ to $0.2 \% ; P=0.2$ ). There was no measurable effect on deep vein thrombosis $(0.8 \% \mathrm{v} 0.6 \%$; $P=0.5)$ or pulmonary embolism $(1.2 \%$ v $1.1 \% ; P=0.9)$.

There were some limitations of our study. It was not a randomised trial and there may have been some imbalance between the groups that we did not account for. The study was unblinded and we compared numerous secondary end points. Although there are likely to be cost benefits of an ERAS program, we did not undertake any health costing analyses.

We found a high level of general acceptance and uptake of the ERAS program and, on the whole, it had a positive effect on patients and staff. We can recommend this orthopaedic ERAS pathway.

Acknowledgements: We thank the research and clinical staff of each of the three hospitals.

Competing interests: This project received funding from the Victorian Department of Health (Chris Potter, Senior Policy Officer). This included a payment to Paul Myles for protocol development, analysis and writing of a report.

Received 22 Apr 2014, accepted 22 Oct 2014.

References are available online at www.mja.com.au. 
1 March LM, Bagga H. Epidemiology of osteoarthritis in Australia. Med J Aust 2004; 180 (5 Suppl): S6-S10.

2 Kehlet $\mathrm{H}$, Mogensen T. Hospital stay of 2 days after open sigmoidectomy with a multimodal rehabilitation programme. Br J Surg 1999; 86: 227-230.

3 Starks I, Wainwright TW, Lewis J, et al. Older patients have the most to gain from orthopaedic enhanced recovery programmes. Age Ageing 2014; 43: 642-548.

4 Larsen K, Hvass KE, Hansen TB, et al. Effectiveness of accelerated perioperative care and rehabilitation intervention compared to current intervention after hip and knee arthroplasty. A before-after trial of 247 patients with a 3-month follow-up. BMC Musculoskelet Disord 2008; 9: 59.

5 Malviya A, Martin K, Harper I, et al. Enhanced recovery program for hip and knee replacement reduces death rate. Acta Orthop 2011; 82: 577-581.

6 Ibrahim MS, Khan MA, Nizam I, Haddad FS. Peri-operative interventions producing better functional outcomes and enhanced recovery following total hip and knee arthroplasty: an evidence-based review. BMC Med 2013; 11: 37.
7 Kehlet H, Søballe K. Fast-track hip and knee replacement - what are the issues? Acta Orthop 2010; 81: 271-272.

8 Husted $\mathrm{H}$, Jensen CM, Solgaard S, Kehlet $\mathrm{H}$. Reduced length of stay following hip and knee arthroplasty in Denmark 2000-2009: from research to implementation. Arch Orthop Trauma Surg 2012; 132: 101-104.

9 Stark PA, Myles PS, Burke JA. Development and psychometric evaluation of a postoperative quality of recovery score: the QoR-15. Anesthesiology 2013; 118: 1332-1340.

10 World Health Organization. International classification of functioning, disability and health. http://www.who.int/classifications/ icf/en (accessed Oct 2014).

1 Mehta RL, Kellum JA, Shah SV, et al. Acute Kidney Injury Network: report of an initiative to improve outcomes in acute kidney injury. Crit Care 2007; 11: R31.

12 Bellomo R, Ronco C, Kellum JA, et al. Acute renal failure - definition, outcome measures, animal models, fluid therapy and information technology needs: the Second International Consensus Conference of the Acute Dialysis Quality Initiative (ADQI) Group. Crit Care 2004; 8: R204-R212.
13 Liu KD, Thompson BT, Ancukiewicz M, et al. Acute kidney injury in patients with acute lung injury: impact of fluid accumulation on classification of acute kidney injury and associated outcomes. Crit Care Med 2011; 39: 2665-2671.

14 Thompson EG, Gower ST, Beilby DS, et al. Enhanced recovery after surgery program for elective abdominal surgery at three Victorian hospitals. Anaesth Intensive Care 2012; 40: 450-459.

15 Husted H, Lunn TH, Troelsen A, et al. Why still in hospital after fast-track hip and knee arthroplasty? Acta Orthop 2011; 82: 679-684.

16 Maessen J, Dejong CH, Hausel J, et al. A protocol is not enough to implement an enhanced recovery programme for colorectal resection. Br J Surg 2007; 94 : 224-231.

17 Scott NB, McDonald D, Campbell J, et al. The use of enhanced recovery after surgery (ERAS) principles in Scottish orthopaedic units - an implementation and follow-up at 1 year, 2010-2011: a report from the Musculoskeletal Audit, Scotland. Arch Orthop Trauma Surg 2013; 133: 117-124.

18 Garson L, Schwarzkopf R, Vakharia S, et al. Implementation of a total joint replacement-focused perioperative surgical home: a management case report. Anesth Analg 2014; 118: 1081-1089. 